\title{
Pain and systemic lupus erythematosus
}

\author{
M. Di Franco', M.P. Guzzo', F.R. Spinelli', F. Atzeni², P. Sarzi-Puttini², \\ F. Conti', C. lannuccelli', \\ ${ }^{1}$ Department of Internal Medicine and Medical Specialties, Rheumatology Unit, \\ La Sapienza University, Rome, Italy \\ ${ }^{2}$ Rheumatology Unit, L. Sacco University Hospital, Milan;
}

\begin{abstract}
Systemic lupus erythematosus (SLE) is an autoimmune disease characterized by heterogeneous clinical manifestations involving virtually the entire body. The pain in SLE can have different causes. The SLE classification criteria include mainly the musculoskeletal manifestations of pain, which are commonly reported as initial symptoms of SLE, such as arthralgia, arthritis and/or myalgia. Chronic widespread pain, which is typical of fibromyalgia (FM), is frequently associated with SLE. The aim of this review is to describe widespread pain and fatigue in SLE, and the association of SLE and FM. Although secondary FM is not correlated with the disease activity, it may interfere with the daily activities of SLE patients. Therefore it is necessary to identify its symptoms and treat them promptly to improve the quality of life of patients. In conclusion, it is essential to identify the origin of pain in SLE in order to avoid dangerous over-treatment in patients with co-existing widespread pain and FM.
\end{abstract}

SUMMARY

Key words: Systemic lupus erythematosus, Fibromyalgia, Chronic widespread pain, Fatigue, Pain.

\section{INTRODUCTION}

Sin hronic widespread pain and fibromyalgia (FM) are frequently associated with rheumatic autoimmune diseases. FM coexists in $30 \%$ of patients with rheumatoid arthritis, $50 \%$ of those with Sjögren syndrome ( $\mathrm{SjS})$, and $30 \%$ of those with systemic lupus erythematosus (SLE) (1). SLE is an autoimmune disease characterized by heterogeneous clinical manifestations involving skin, joints, kidneys, heart, nervous system and virtually any other organ in the body.

Its association with FM, which is a syndrome characterized by chronic widespread pain and symptoms such as fatigue and sleep disturbances, can sometimes complicate the evaluation of pain, which should be differentiated from SLE-related disease activity or chronic damage. The most widely used indices to measure objectively the disease activity in SLE are the British isles lupus assessment group (BILAG) index, the European consensus lupus activity measurement (ECLAM), and the systemic lupus erythematosus disease activity index (SLEDAI), none of which are specifically meant to evaluate musculoskeletal pain per se. The health-related quality of life (HRQoL) of SLE patients can be assessed by means of questionnaires, such as the short form 36 (SF-36) (2), and the patient acceptable symptom state, which is a simple questionnaire to assess well-being in patients with various rheumatic conditions, including SLE (3).

The classification criteria started to be developed already in the early 1970s, where the criteria currently in use were introduced by the American College of Rheumatology (ACR) in 1982, and revised in 1997 (4). In 2012, the Systemic Lupus International Collaborating Clinics (SLICC) group proposed a new set of criteria that also include less specific clinical manifestations that are frequently reported by SLE patients (5), with pain being mainly confined to the musculoskeletal system. The aim of this review is to describe widespread pain and fatigue in SLE, and the association of SLE and FM. $\overline{\text { Corresponding author: }}$ Manuela Di Franco Department of Internal Medicine and Medical Specialties

Rheumatology Unit La Sapienza University, Rome, Italy E-mail: manuela.difranco@uniroma1.it 


\section{PATHOGENESIS}

Pain in SLE can be attributable to various causes (e.g. inflammatory, neuropathic, and central pain), although it is most often associated with joint inflammation. The ACR SLE classification criteria refer to the presence of synovitis in two or more joints (characterized by swelling or effusion), whereas the proposed SLICC criteria extend the definition of musculoskeletal involvement to inflammatory arthralgia (tenderness in two or more joints with morning stiffness lasting at least 30 minutes) $(4,5)$. Joint manifestations can precede other SLE features, and typically consist in arthralgia and/or non-erosive, non-deforming arthritis. However, in same cases, arthritis can lead to reducible deformities, known as Jaccoud's arthropathy (6).

Like in other inflammatory joint diseases, the pathogenic mechanisms of inflammatory arthritis in SLE involve the production of cytokines such as interleukin (IL)-6, IL-17, interferon- $\alpha$, IL-18, tumor necrosis factor- $\alpha$ and B lymphocyte stimulator. These inflammatory mediators play a crucial role in the differentiation, maturation and activation of the immunocompetent cells involved in the local inflammatory process and tissue injury (7).

Another source of pain is lupus headache, which is reported by $32-66 \%$ of SLE patients and can have various causes, including neuropsychiatric SLE (NPSLE) (8-10). In NPSLE patients, a close association was identified between cognitive impairment and depression, pain and fatigue, but the underlying causes are still unclear. Autoantibodies cross-reacting with DNA and $\mathrm{N}$-methyl-D-aspartate receptors, anti-endothelial antibodies and anti-phospholipid antibodies are the most common factors associated with the pathogenesis of NPSLE $(11,12)$. Depression, pain, and fatigue may also sometimes reflect an underlying central nervous system (CNS) disorder (13). Abdominal pain is reported by $8-40 \%$ of SLE patients (14), and may due to various causes, including mesenteric vasculitis and pancreatitis (15). The association of pain and cutaneous lupus has been recently evaluated and it was found that the pain was more intense in patients with vasculitic and bullous lesions, followed by patients with oral ulcers (16).

One of the first symptoms reported by SLE patients is pain resulting from musculoskeletal involvement (17), which affects approximately $50-90 \%$ of patients during the course of the disease (18).

Chronic widespread pain, which affects $5-10 \%$ of the general population (19), is reported by $65-80 \%$ of SLE patients (20, 21). Central sensitization, characterized by an enhanced peripheral nociceptive input and abnormal central pain processing of the CNS, seems to be the pathogenic mechanism causing hyperalgesia and allodynia (22). Fatigue is the most common constitutional symptom associated with pain in SLE patients (23) and has a prevalence ranging from $50 \%$ to over $80 \%$ (24). It can also be the most debilitating symptom $(25,26)$, although it is not associated with the disease activity or the chronic organ damage, but only with anemia.

\section{PUBLISHED FINDINGS}

Chronic widespread pain is the cornerstone of FM, which is characterized by tenderness on palpation of at least 11/18 tender points (TPs) (27). FM is also characterized by fatigue and multiple associated symptoms, such as sleep, mood and neurocognitive disorders, as indicated by the 2010 ACR classification criteria (28).

A number of studies investigated the prevalence of FM in SLE patients, and evaluated how concomitant FM can influence the symptoms and the activity of SLE. The prevalence of FM varies depending on the ethnic group suggesting a possible influence of geographical, psychological and socio-cultural factors. Its prevalence was found to be $8.2 \%$ in a cohort of Indian SLE patients, 9.5\% in Mexican SLE patients, $10 \%$ in a Spanish cohort, and $25 \%$ among Turkish SLE patients (29-32).

Various authors studied the prevalence of FM in LES patients. Morand et al. reported a $25.3 \%$ prevalence in a cohort of 
87 SLE patients (33), while Middleton et al. reported a $22 \%$ prevalence in a group of 102 SLE patients (34). However, although $23 \%$ of the patients in the latter study presented widespread pain, they did not meet the 1990 ACR classification criteria. The authors reported that SLE patients with concomitant FM have significantly more frequent and severe symptoms, but no significant difference in SLE activity measures was identified between patients with or without FM (34).

Similarly, the presence of FM in $21 \%$ of a Canadian cohort of 119 SLE patients did not correlate with the disease activity and the damage indices, but it was closely correlated with the quality of life which was measured on the basis of the SF-36 questionnaire (35). Friedman et al. confirmed that there was no correlation between the clinical scores of disease activity, disease damage, specific organ dysfunction and concomitant FM, which was diagnosed only in 5\% of SLE patients (36).

Taylor et al. investigated whether FM was more frequent in patients who complained fatigue, and found that only $10 \%$ of these patients fulfilled the criteria for FM (37).

An Israeli group found that SLE patients with FM had significantly lower HRQoL scores than those without FM (38). In 2005, Akkasilpa et al. evaluated the association between tender point positivity and health status in a group of 173 SLE patients using the health assessment questionnaire (HAQ): $17.3 \%$ of the patients had $\geq 11$ TPs and the number of TPs was significantly associated with their HAQ scores (39).

We have recently analyzed the prevalence of widespread pain and fatigue in a group of Italian patients with SLE or SjS. FM was diagnosed in a significantly higher percentage of SLE patients (33\% vs 18\%). Moreover, the SLE patients showed a correlation between HAQ scores and fatigue and pain, whereas no correlation was found between the associated FM and the disease activity indices (21).

Grafe et al. observed that FM was more frequent in SLE patients (prevalence of $16.7 \%$ ) than in patients with cutaneous lupus. The patients with SLE and concomi- tant FM also reported significantly more often symptoms such as headache, morning stiffness, diffuse alopecia, and arthralgia (40).

Pain is associated with perceptions of reduced physical functioning and coping resources, and higher levels of anxiety and depression. Consequently SLE patients need psychosocial interventions in order to relieve pain and distress and improve coping skills (41). Pain coping cognitions, such as self-efficacy for pain control and pain catastrophizing, may influence symptoms such as pain, stiffness, fatigue, and psychological distress in SLE patients. Recent data demonstrates that patients with low levels of self-efficacy for pain control and/or high levels of pain catastrophizing reported more physical symptoms and psychological distress, and highlights the importance of assessing coping constructs in SLE patients (42).

Apart from FM, pain can interfere with the quality of life, and the SLE patients who experience the highest levels of pain also report stiffness and fatigue. Pain can also cause functional limitations that lead to a significant economic burden on the society as a whole (43). The working productivity of SLE patients with the highest levels of pain is lower than that of SLE patients with less pain (44), and pain also predicted negative energy levels (45). Pettersson et $a l$. found that the most frequently reported symptoms by SLE patients are fatigue, pain and musculoskeletal distress, all of which are related to low HRQoL scores, although only fatigue has an impact on both mental and physical components (46).

Table I - Common symptoms of systemic lupus erythematosus and fibromyalgia.

\begin{tabular}{|l|}
\hline Musculoskeletal pain \\
\hline Fatigue \\
\hline Stiffness \\
\hline Sicca symptoms \\
\hline Cold-induced vasospasm \\
\hline Cognitive dysfunction \\
\hline Depression \\
\hline Headache \\
\hline Abdominal pain \\
\hline
\end{tabular}


The differential diagnosis of SLE and FM may be difficult because the two diseases have some symptoms in common (Tab. I). In a recent study, 15 patients with FM had been previously misdiagnosed as having connective tissue diseases, including SLE (47). Low antinuclear antibody (ANA) titers are commonly reported in both the general population and FM patients, and so it is necessary to evaluate the clinical data in order to avoid misdiagnoses. Wallace et al. described a group of 44 patients referred for a rheumatologic visit because of pain and ANA positivity in order to rule out lupus. After six months of follow-up, 19 (43\%) fulfilled the ACR criteria for SLE, and 14 (32\%) fulfilled the ACR criteria for FM (48). Although secondary FM is not associated with the disease activity, it may worsen the quality of life of patients with SLE, who may experience increased fatigue because of FM. Moreover, chronic fatigue without FM can influence their HRQoL even if it is not related to SLE disease activity or organ damage (49). A recent study has confirmed that SLE patients have lower SF-36 scores than the general population; FM is independently associated with a poorer HRQoL, underlining the need to treat pain promptly and accurately (50).

\section{THERAPY}

The treatment of pain in SLE patients requires a differential diagnosis. In particular, it is necessary to establish whether pain is localized or widespread, visceral or musculoskeletal. The first step is to evaluate the SLE activity, and optimize a specific SLE treatment with immunosuppressive drugs and steroids $(51,52)$.

FM symptoms in an SLE patient may be misinterpreted as lupus disease activity, thus leading to over-treatment. Once it is excluded that the cause of pain is not attributable to the disease activity, the same treatment used for FM can be administered. Analgesic drugs, such as acetaminophen or tramadol, can be safely used in patients with widespread pain and SLE (53). Anti-epileptic drugs, such as gabapentin and pregabalin, have been approved for the treatment of neuropathic pain. Pregabalin is also approved by the American Food and Drug Administration (FDA) for FM. Both drugs have a favorable safety and tolerability profile (54). Low doses of tricyclic antidepressants are useful in the case of musculoskeletal pain and headache. Selective serotonin reuptake inhibitors and serotonin norepinephrine reuptake inhibitors, such as duloxetine (approved by the FDA for FM), can be used in SLE patients with pain and depression (55). In many cases non-pharmacological treatments, such as aerobic exercise and cognitive behavioral therapy, may also be useful (56).

In conclusion, an accurate diagnosis of musculoskeletal, inflammatory and SLErelated or FM-associated pain is essential in order to start the right therapy and avoid dangerous over-treatment in SLE patients with co-existing widespread pain and FM.

\section{REFERENCES}

1. Atzeni F, Cazzola M, Benucci M, Di Franco M, Salaffi F, Sarzi-Puttini P. Chronic widespread pain in the spectrum of rheumatological diseases. Best Pract Res Clin Rheumatol. 2011; 25: 165-71.

2. Griffiths B, Mosca M, Gordon C. Assessment of patients with systemic lupus erythematosus and the use of lupus disease activity indices. Best Pract Res Clin Rheumatol. 2005; 19: 685-708.

3. Conti F, Ceccarelli F, Massaro L, Pacucci VA, Miranda F, Truglia S, et al. Evaluation of the patient acceptable symptom state (PASS) in Italian patients affect by systemic lupus erythematosus: association with disease activity indices. PLoS One. 2013; 8: e73517.

4. Hochberg MC. Updating the American College of Rheumatology revised criteria for the classification of systemic lupus erythematosus. Arthritis Rheum. 1997; 40: 1725.

5. Petri M, Orbai AM, Alarcón GS, Gordon C, Merrill JT, Fortin PR, et al. Derivation and validation of the Systemic Lupus International Collaborating Clinics classification criteria for systemic lupus erythematosus. Arthritis Rheum. 2012; 64: 2677-86.

6. Skare TL, Godoi Ade L, Fereira VO. Jaccoud arthropathy in systemic lupus erythematosus: clinical and serological findings. Rev Assoc Med Bras. 2012; 58: 489-92. 
7. Yap DY, Lai KN. The role of cytokines in the pathogenesis of systemic lupus erythematosus - from bench to bedside. Nephrology. 2013; 18: 243-55.

8. Sfikakis PP, Mitsikostas DD, Manoussakis MN, Foukaneli D, Moutsopoulos HM. Headache in systemic lupus erythematosus: a controlled study. Br J Rheumatol. 1998; 37: 300-3.

9. Glanz BI, Venkatesan A, Schur PH, Lew RA, Khoshbin S. Prevalence of migraine in patients with systemic lupus erythematosus. Headache. 2001; 41: 285-9.

10. Omdal R, Waterloo K, Koldingsnes W, Husby G, Mellgren SI. Somatic and psychological features of headache in systemic lupus erythematosus. J Rheumatol. 2001; 28: 772-9.

11. Conti F, Alessandri C, Perricone C, Scrivo R, Rezai S, Ceccarelli F, et al. Neurocognitive dysfunction in systemic lupus erythematosus: association with antiphospholipid antibodies, disease activity and chronic damage. PLoS One. 2012; 7: e33824.

12. Conti F, Alessandri C, Bompane D, Bombardieri M, Spinelli FR, Rusconi AC, et al. Autoantibody profile in systemic lupus erythematosus with psychiatric manifestations: a role of anti-endothelial-cell-antibodies. Arthritis Res Ther. 2004; 6: R366-72.

13. Kozora E, Ellison MC, West S. Depression, fatigue, and pain in systemic lupus erythematosus (SLE): relationship to the American College of Rheumatology SLE neuropsychological battery. Arthritis Rheum. 2006; 55: 628-35.

14. Kwok SK, Seo SH, Ju JH, Park KS, Yoon $\mathrm{CH}$, Kim WU, et al. Lupus enteritis: clinical characteristics, risk factor for relapse and association with anti-endothelial cell antibody. Lupus. 2007; 16: 803-9.

15. Yuan S, Lian F, Chen D, et al. Clinical features and associated factors of abdominal pain in systemic lupus erhitematosus. J Rheumatol. 2013; 40: 2015-22.

16. Méndez-Flores S, Orozco-Topete R, Bermúdez-Bermejo P, Hernández-Molina G. Pain andpruritus in cutaneous lupus: their association with dermatologic quality of life and disease activity. Clin Exp Rheumatol. 2013; 31: 940-2.

17. Ozbek S, Sert M, Paydas S, Soy M. Delay in the diagnosis of SLE: the importance of arthritis/arthralgia as the initial symptom. Acta Med Okayama. 2003; 57: 187-90.

18. Waldheim E, Elkan AC, Pettersson S, van Vollenhoven R, Bergman S, Frostegård J, et al. Health-related quality of life, fatigue and mood in patients with SLE and high levels of pain compared to controls and patients with low levels of pain. Lupus. 2013; 22: 1118-27.

19. Sarzi-Puttini P, Atzeni F, Mease P. Chronic widespread pain or fibromyalgia? That is the question. Best Pract Res Clin Rheumatol. 2011; 25: 131-2.

20. Wolfe F, Petri M, Alarcón Gs, Goldman J, Chakravarty EF, Katz RS, et al. Fibromyalgia, systemic lupus erythematosus (SLE), and evaluation of SLE activity. J Rheumatol. 2009; 36: 82-8.

21. Iannuccelli C, Spinelli FR, Guzzo MP, Priori R, Conti F, Ceccarelli F, et al. Fatigue and widespread pain in systemic lupus erythematosus and Sjögren's syndrome: symptoms of the inflammatory disease or associated fibromyalgia? Clin Exp Rheumatol. 2012; 30: 117-21.

22. Yunus MB. Central sensitivity syndrome: a new paradigm and group nosology for fibromyalgia and overlapping conditions, and the related issue of disease versus illness. Semin Arthritis Rheum. 2008; 37: 339-52.

23. Petri M, Kawata AK, Fernandes AW, Gajria K, Greth W, Hareendran A, et al. Impaired health status and the effect of pain and fatigue on functioning in clinical trial patients with systemic lupus erythematosus. J Rheumatol. 2013; 40: 1865-74.

24. Tench CM, McCurdie I, White PD, D’Cruz DP. The prevalence and associations of fatigue in systemic lupus erythematosus. Rheumatology (Oxford). 2000; 39: 1249-54.

25. McKinley PS, Ouellette SC, Winkel GH. The contributions of disease activity, sleep patterns, and depression to fatigue in systemic lupus erythematosus. A proposed model. Arthritis Rheum. 1995; 38: 826-34.

26. Wysenbeek AJ, Leibovici L, Weinberger A, Guedj D. Fatigue in systemic lupus erythematosus. Prevalence and relation to disease expression. Br J Rheumatol. 1993; 32: 633-5.

27. Wolfe F, Smythe HA, Yunus MB, Bennett RM, Bombardier C, Goldenberg DL, et al. The American College of Rheumatology 1990 criteria for the classification of fibromyalgia: report of the Multicenter Criteria Committee. Arthritis Rheum. 1990; 33: 160-72.

28. Wolfe F, Clauw DJ, Fitzcharles MA, Goldenberg DL, Katz RS, Mease P, et al. The American College of Rheumatology preliminary diagnostic criteria for fibromyalgia and measurement. Arthritis Care Res (Hoboken). 2010; 62: 600-10.

29. Handa R, Aggarwal P, Wali JP, Wig N, Dwivedi SN. Fibromyalgia in Indian patients with SLE. Lupus. 1998; 7: 475-8.

30. Valencia-Flores M, Cardiel MH, Santiago V, Resendiz M, Castaño VA, Negrete O, et al. Prevalence and factors associated with fibromyalgia in Mexican patients with systemic lupus erythematosus. Lupus. 2004; 13 : 4-10.

31. López-Osa A, Jiménez-Alonso J, GarcíaSánchez A, Sánchez-Tapia C, Pérez M, Peralta MI, et al. Fibromyalgia in Spanish patients 
with systemic lupus erythematosus. Lupus. 1999; 8: 332-3.

32. Karaaslan Y, Oztürk M, Haznedaroğlu S. Secondary fibromyalgia in Turkish patients with rheumatologic disorders. Lupus. 1999; 8: 486.

33. Morand EF, Miller MH, Whittingham S, Littlejohn GO. Fibromyalgia syndrome and disease activity in systemic lupus erythematosus. Lupus. 1994; 3: 187-91.

34. Middleton GD, Mcfarlin JE, Lipsky PE. The prevalence and clinical impact of fibromyalgia in systemic lupus erythematosus. Arthritis Rheum. 1994; 37: 1181-8.

35. Gladman DD, Urowitz MB, Gough J, MacKinnon A. Fibromyalgia is a major contributor to quality of life in lupus. J Rheumatol. 1997; 24: 2145-8.

36. Friedman AW, Tewi MB, Ahn C, McGwin G Jr, Fessler BJ, Bastian HM, et al. Systemic lupus erythematosus in three ethnic groups: XV. Prevalence and correlates of fibromyalgia. Lupus. 2003; 12: 274-9.

37. Taylor J, Skan J, Erb N, Carruthers D, Bowman S, Gordon C, et al. Lupus patients with fatigue - is there a link with fibromyalgia syndrome? Rheumatology. 2000; 39: 620-3.

38. Abu-Shakra M, Mader R, Langevitz P, Friger M, Codish S, Neumann L, et al. Quality of life in systemic lupus erythematosus: a controlled study. J Rheumatol. 1999; 26: 306-9.

39. Akkasilpa S, Goldman D, Magder LS, Petri M. Number of fibromyalgia tender points is associated with health status in patients with systemic lupus erythematosus. J Rheumatol. 2005; 32: 48-50.

40. Gräfe A, Wollina U, Tebbe B, Sprott H, Uhlemann C, Hein G. Fibromyalgia in lupus erythematosus. Acta Derm Venereol. 1999; 79: 62-4.

41. Greco CM, Rudy TE, Manzi S. Adaptation to chronic pain in systemic lupus erythematosus: applicability of the multidimensional pain inventory. Pain Med. 2003; 4: 39-50.

42. Somers TJ, Kurakula PC, Criscione-Schreiber L, Keefe FJ Clowse ME. Self-efficacy and pain catastrophizing in systemic lupus erythematosus: relationship to pain, stiffness, fatigue, and psychological distress. Arthritis Care Res (Hoboken). 2012; 64: 1334-40.

43. Bliddal H, Danneskiold-Samsøe B. Chronic widespread pain in the spectrum of rheumatological diseases. Best Pract Res Clin Rheumatol. 2007; 21: 391-402.

44. Clarke AE, Bloch DA, Danoff DS, Esdaile JM. Decreasing costs and improving outco- mes in systemic lupus erythematosus: using regression trees to develop health policy. J Rheumatol. 1994; 21: 2246-53.

45. Moldovan I, Cooray D, Carr F, Katsaros E, Torralba K, Shinada S, et al. Pain and depression predict self-reported fatigue/energy in lupus. Lupus. 2013; 22: 684-9.

46. Pettersson S, Lövgren M, Eriksson LE, Moberg C, Svenungsson E, Gunnarsson I, et al. An exploration of patient-reported symptoms in systemic lupus erythematosus and the relationship to health-related quality of life. Scand J Rheumatol. 2012; 41: 383-90.

47. Di Franco M, Iannuccelli C, Bazzichi L, Atzeni F, Consensi A, Salaffi F, et al. Misdiagnosis in fibromyalgia: a multicentre study. Clin Exp Rheumatol. 2011; 29: S104-8.

48. Wallace DJ, Schwartz E, Chi-Lin H, Peter JB. The 'rule out lupus' rheumatology consultation: clinical outcomes and perspectives. J Clin Rheumatol. 1995; 1: 158-64.

49. Kiani AN, Petri M. Quality-of-life measurements versus disease activity in systemic lupus erythematosus. Curr Rheumatol Rep. 2010; 12: 250-8.

50. Kiani AN, Strand V, Fang H, Jaranilla J, Petri M. Predictors of self-reported health-related quality of life in systemic lupus erythematosus. Rheumatology (Oxford). 2013; 52: 1651-7.

51. Bertsias G, Ioannidis JP, Boletis J, Bombardieri S, Cervera R, Dostal C, et al. EULAR recommendations for the management of systemic lupus erythematosus. Report of a Task Force of the EULAR Standing Committee for International Clinical Studies Including Therapeutics. Ann Rheum Dis. 2008; 67: 195-205.

52. Conti F, Ceccarelli F, Massaro L, Cipriano E, Di Franco M, Alessandri C, et al. Biological therapies in rheumatic diseases. Clin Ter. 2013; 164: e413-28.

53. Bennett RM, Schein J, Kosinski MR, Hewitt DJ, Jordan DM, Rosenthal NR. Impact of fibromyalgia pain on health-related quality of life before and after treatment with tramadol/ acetaminophen. Arthritis Rheum. 2005; 53: 519-27.

54. Spaeth M. Is pregabalin a safe and effective treatment for patients with fibromyalgia? Nat Clin Pract Rheumatol. 2008; 4: 514.

55. Mease PJ, Dundon K, Sarzi-Puttini P. Pharmacotherapy of fibromyalgia. Best Pract Res Clin Rheumatol. 2011; 25: 285-97.

56. Di Franco M, Iannuccelli C, Atzeni F, Cazzola M, Salaffi F, Valesini G, et al. Pharmacological treatment of fibromyalgia. Clin Exp Rheumatol. 2010; 28: S110-6. 